\title{
Research on Spectrum Resources Spatial Reuse Algorithm Based on Game Theory in Cognitive Radio
}

\author{
Fulai Liu ${ }^{1}$, Zhenxing Sun ${ }^{2,4, ~}$, Ruiyan $\mathrm{Du}^{1}$, Lei $\mathrm{Shi}^{3}$ \\ ${ }^{1}$ Engineering Optimization \& Smart Antenna Institute, Northeastern University at Qinhuangdao, Qinhuangdao, China \\ ${ }^{2}$ School of Computer Science and Engineering, Northeastern University, Shenyang, China \\ ${ }^{3}$ Spreadtrum Communications Company Limited, Shanghai, China \\ ${ }^{4}$ Northeast Petroleum University at Qinhuangdao, Qinhuangdao, China

\section{Email address:} \\ fulailiu@126.com (Fulai Liu), sunzx-0@163.com (Zhenxing Sun), ruiyandu@126.com (Ruiyan Du), 55577519@qq.com (Lei Shi) \\ ${ }^{*}$ Corresponding author
}

\section{To cite this article:}

Fulai Liu, Zhenxing Sun, Ruiyan Dui, Lei Shi. Research on Spectrum Resources Spatial Reuse Algorithm Based on Game Theory in Cognitive Radio. Science Discovery. Vol. 5, No. 5, 2017, pp. 355-361. doi: 10.11648/j.sd.20170505.19

Received: July 2, 2017; Accepted: August 4, 2017; Published: August 17, 2017

\begin{abstract}
In cognitive radio system, spectrum reuse is one of the main methods to improve the utilization of spectrum resources. Nevertheless, it has been unable to further improve spectrum efficiency that the reuse is implemented exclusively from the dimension of frequency. Taking account of the problem of spectrum resource spatial reuse in addition to the reuse of frequency, and aiming at the problem of the network transmission power optimization based on spectrum resource spatial reuse method in cognitive network, this paper proposes a spectrum resource spatial reuse algorithm based on Game Theory. Game theory is used by the algorithm to establish a game model of spectrum resources spatial reuse. A price function based on the channel quality is introduced to ensure the fairness of cognitive user to allocate power in each channel. A successive over relaxation iteration algorithm is used to solve the Nash equilibrium. Simulation results confirm that the proposed algorithm not only has high reliable detection performance to reduce the total transmission power, but also can ensure the service quality of cognitive users.
\end{abstract}

Keywords: Cognitive Radio, Spatial Reuse, Game Theory, Power Allocation

\section{认知无线电系统中基于博弯论的频谱资源空域重用算法研究}

\author{
刘福来 ${ }^{1}$, 孙振兴 ${ }^{2}$ 4* $^{*}$ 杜瑞燕 ${ }^{1}$, 石否 $^{3}$ \\ ${ }^{1}$ 工程优化与智能天线研究所, 东北大学秦皇岛分校, 秦皇岛, 中国 \\ ${ }^{2}$ 计算机科学与工程学院, 东北大学, 沈阳, 中国 \\ 展讯通信有限公司, 上海, 中国 \\ 4东北石油大学秦皇岛分校, 秦皇岛, 中国
}

邮箱

fulailiu@126.com（刘福来）, sunzx-0@163.com（孙振兴）, ruiyandu@126.com（杜瑞燕）,55577519@qq.com（石否）

摘要: 在认知无线电系统中进行频谱重用是提高频谱资源利用率的核心手段, 然而单一进行频域重用已无法进一步提 升频谱效率, 本文在频域重用基础上考虑频谱资源的空域重用问题, 并针空域重用中网络传输总功率优化问题, 提出 了一种基于博亦论的频谱资源空域重用算法。利用博亦论建立了频谱资源空域重用问题的博恋模型, 然后基于认知用 户的信道质量引入价格因子来保证认知用户在各信道上分配功率的公平性。最后利用逐次松驰迭代算法求解纳什均衡 
点, 获得了功率分配的最优解。仿真结果证实所提算法不仅能够实现网络传输总功率最小化, 而且能够保证认知用户 的服务质量。

关键词: 认知无线电, 空域重用, 博弯论, 功率分配

\section{1. 引言}

随着无线通信技术的飞速发展, 新的无线通信业务层 出不穷, 用户数量不断增加, 人们对无线频谱资源的需求 也在日益增长。然而, 大部分可用频谱资源已经分配殆尽, 频谱资源短缺问题已成为未来无线通信技术发展的阻碍。 针对日益增长的无线通信需求和频谱资源短缺之间的矛 盾, 有些组织和机构不得不在更高的频段上开展比如链路 自适应技术、多天线技术等新技术的研究。另外, 对现有 频谱资源利用率不高也是造成频谱资源紧张的主要原因 之一。为了提高频谱资源利用率, 缓解频谱资源紧张的现 状, 认知无线电技术引起了人们的广泛关注 [1]。

在认知无线电系统中, 由于分配给授权用户的频段通 常会在某一时段或某一空间区域内未被授权用户占用, 从 而形成频谱空穴，所以频谱空穴具有时间、频率、空间等 多个维度。因此, 从多个维度联合对授权频谱资源进行重 用是认知无线电未来的发展方向 [2]。

近年来, 频谱资源的空域重用问题受到了专家学者们 的广泛关注。2006年来自艾莉森公司的P.W. 登特设计了 可以从单根公共天线发射多个频段无线电信号的发射机, 并申请了专利, 这一专利的申请使得相同的信源信号经过 不同的高频载波调制之后可以从一个公共的天线上发射 出去。该专利所设计的发射机需要装备多个调制器, 每个 调制器至少能够生成一个固定幅度、相位被调的驱动信号, 专利中的发射机至少还需要一个能够根据所调制驱动信 号的变化而自适应进行配置的饱和功率放大器, 一个耦合 网络用于将以上所述饱和功率放大器的输出端串联起来, 产生组合信号经过公共天线进行发射 [3], 专利中所申请的 发射机为认知无线电频谱资源空域重用技术的实现提供 了可能。文献[4]中作者针对频谱资源空域重用问题, 利用 波束形成技术，设计了一种频谱资源空域重用的方案，该 方案中认知无线电设备需要装备天线阵列进行通信, 当认 知用户通信的发送端 $\mathrm{A}$ 和接收端B之间存在授权用户时, 认知用户仍可通过中继节点来实现通信, 此时只要网络中 存在一个结点 $\mathrm{C}$, 并且保证从发送结点 $\mathrm{A}$ 到 $\mathrm{C}$ 之间和从接收 节点 $\mathrm{B}$ 到 $\mathrm{C}$ 之间不存在授权用户即可, 这样的中继结点在 现实生活的网络中是容易找到的, 因此, 该方案从频域和 空域两个维度联合进行频谱资源分配，达到了在不同空间 位置的认知用户和授权用户能够共享同一频谱资源的目 的。文献[5]针对WRAN小区内认知基站与授权无线麦克风 用户之间和认知无线电用户与授权用户之间空-频二维干 扰问题, 建立了一种新的WRAN与授权用户的共存模型, 以最大化系统下行速率为目标, 给出了WRAN单小区中信 道分配的数学模型, 文中针对求解复杂度较高的问题, 基 于图论模型将信道等效为二分图中的最大匹配问题, 运用 匈牙利算法求得了问题的最优解, 解决了WRAN小区内频
谱资源空域重用问题。文献[6]中针对MISO认知无线电网 络中资源空域重用问题，在不完备信道状态信息交互的情 况下, 利用波束形成技术, 解决了功率最小化问题, 并有 效地控制了对主用户的干扰, 但是文献[6]中的所提方法仅 仅是针对单频段的情况, 将此方法应用到多频段认知无线 电系统中并不能取得理想的效果。在多频段MIMO认知无 线电网络的研究领域, 文献[7]中考虑了认知用户速率最大 化问题, 通过构建非协作博弯模型, 提出了分布式算法, 求得认知无线电系统网络的纳什均衡点。文献 [8]针对电视 频段范围内的认知无线电系统频谱资源空域重用问题, 构 建了一种地理位置数据库 (Geo-location Database), 用以 解决以电视频段为授权频段网络中的认知用户和多种授 权用户（TV发射塔、无线麦克风塔等）共存的问题，从 实际角度出发解决了认知无线电系统频谱资源空域重用 问题。文献[9]中针对频谱资源空域重用中对授权用户造成 的干扰问题，提出了利用多天线技术改善认知无线电系统 的方案, 从空间相关性角度出发, 直接在干扰温度模型中 引入空域参数, 在频-空二维空间内实现了对认知用户进 行功率分配的目的。文献 [10]提出了一种更为实用的空时 域结合频谱共享方案, 给出了时域频谱共享区域的上边界 和自由空域频谱共享区域的下边界。针对现有的静态频谱 管理机制导致频谱资源在空间维度和时间维度上的低利 用率，文献[11]将异质频谱的重分配问题建模成组合拍卖 模型, 实现了防策略性和近似社会福利最大化。在文献[12] 中, 作者针对网络总功率消耗优化问题, 构建了联合功率、 频率分配和波束优化数学模型, 并且证明了纳什均衡点的 存在性, 这也为本文的研究提供了依据。

本文针对频谱资源空域重用中网络传输总功率优化 问题, 提出一种基于博弯论的频谱资源空域重用算法, 通 过利用博弯论建立频谱资源空域重用问题的博弯模型, 然 后基于认知用户的信道质量引入价格因子来保证认知用 户在各信道上分配功率的公平性, 通过求解频谱资源空域 重用问题的纳什均衡点, 获得功率分配的最优解。本文首 先给出频谱资源空域重用的数据模型, 然后详细阐述所提 算法的原理, 最后通过仿真实验验证所提算法的有效性。

\section{2. 数据模型}

假设网络模型为分布式的认知无线电MISO网络, 如 图1所示, 整个认知网络包括 $N$ 条认知用户通信链路, 每 条通信链路中认知用户的接收端和授权用户的接收端都 只装备一根天线接收信号, 发射端装备 $M$ 根天线阵列利 用波束形成技术进行通信，所有认知用户发射端都能够同 时在 $K$ 个正交子信道上发送信息,各个正交子信道的中心 
频率分别设为 $f_{1}, f_{2}, \ldots, f_{K}, \Phi_{N}=\{1,2, \ldots, \mathrm{N}\}$ 为认知用户 链路集合, 可用的授权子信道集合定义为 $\Psi_{K}=\{1,2, \ldots, \mathrm{K}\}$ 。 其中在第 $i$ 个认知用户的通信链路中, 发射端在信道 $f_{k}$ 上 发送的信源符号为 $b_{i}^{(k)}, b_{i}^{(k)} \in C^{1 \times 1}$, 在第 $k$ 个信道上认知
用户 $i$ 的发送端预编码向量为 $\boldsymbol{w}_{i}^{(k)} \in C^{M \times 1}$, 即认知用户 $i$ 的发射端通过 $M$ 根阵列天线在第 $k$ 个信道上向其接收端 实际传输的符号向量为 $\boldsymbol{x}_{i}^{k}=\boldsymbol{w}_{i}^{(k)} b_{i}^{(k)}$ 。

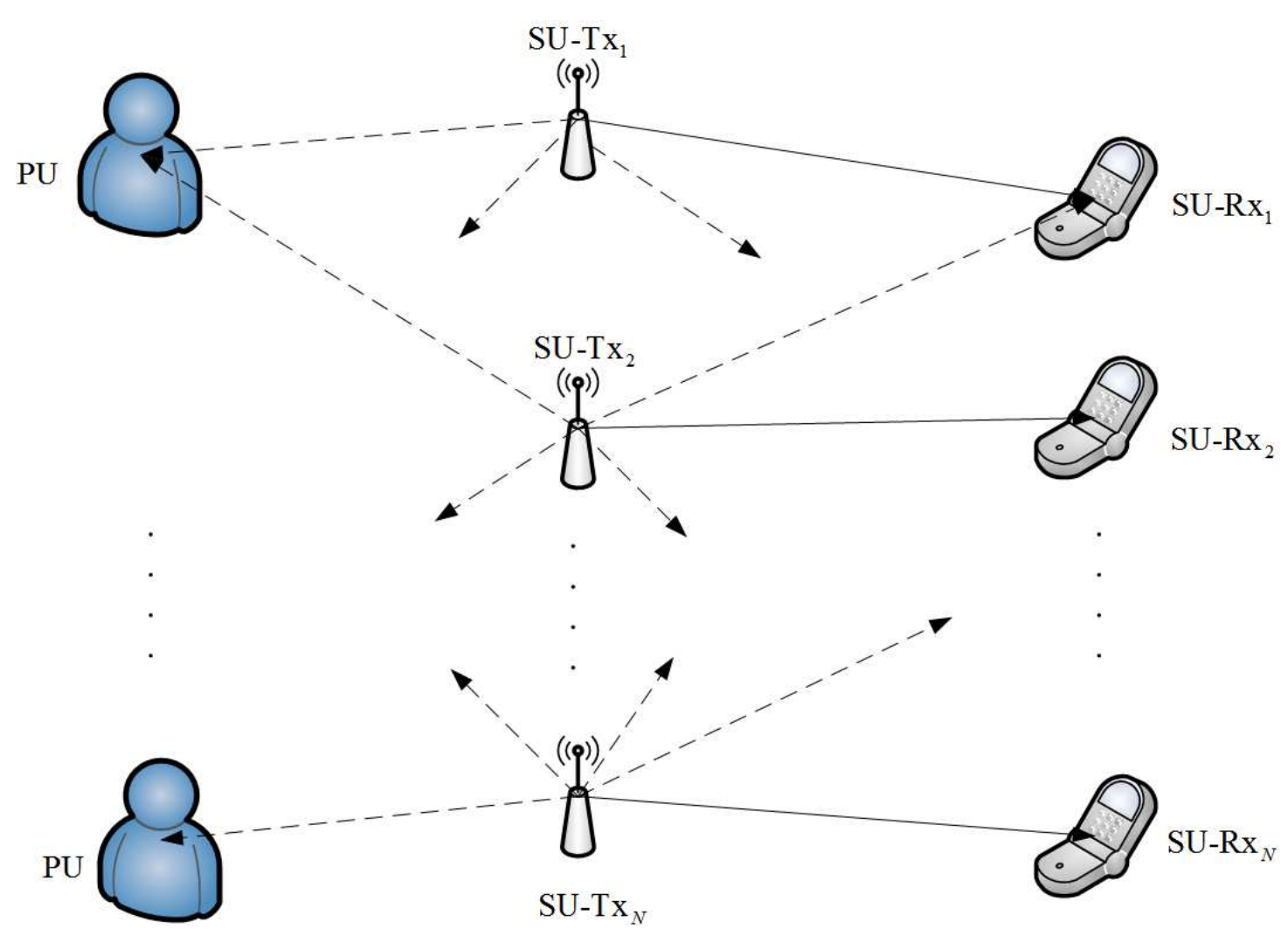

图1 分布式网络模型。

为不失一般性, 假设信源符号 $b_{i}^{(k)}$ 满足 $E\left\{\left.b_{i}^{k}\right|^{2}\right\}=1$, $E\left\{b_{i_{1}}^{\left(k_{1}\right)} b_{i_{2}}^{\left(k_{2}\right)}\right\}=0$, 并且各个认知用户在不同的信道上传输 的信源符号不同, 即 $\forall i_{1}, i_{2} \in \Phi_{N}, \forall k_{1}, k_{2} \in \Psi_{K}$, $\left(i_{1}, k_{1}\right) \neq\left(i_{2}, k_{2}\right)$ 。认知用户 $i$ 的接收端在第 $k$ 个信道上接收 的信号可以表示为:

$$
y_{i}^{(k)}=\boldsymbol{h}_{i, i}^{(k)} \boldsymbol{x}_{i}^{(k)}+\sum_{j \in \Psi_{K} \backslash\{k\}} \boldsymbol{h}_{i, j}^{(k)} \boldsymbol{x}_{j}^{(k)}+n_{i}^{(k)}
$$

其中 $\boldsymbol{h}_{i, j}^{(k)} \in C^{1 \times M}$ 表示认知用户 $j$ 到认知用户 $i$ 之间的 第 $k$ 个信道的信道增益, $n_{i}^{(k)}$ 表示认知用户 $i$ 在信道 $f_{k}$ 上 传输信息时受到的干扰, 服从均值为零, 方差为 1 的高斯 分布。假设认知用户受到的干扰包括网络的背景噪声和授 权用户通信产生的干扰。

\section{3. 算法原理}

\section{1 . 问题描述}

在认知无线电MISO网络中, 认知网络为保证授权用 户的通信质量不受到认知用户的影响, 规定认知用户对授
权用户所造成的干扰应该控制在一个合理的容限值之内, 认知网络为解决授权用户干扰容限值的设定问题, 设定统 一的用户接入机制, 为各个授权用户在不同的子信道上设 立不同的干扰容限, 认知用户在各个信道上的传输功率之 和不得超过此干扰容限值, 定义 $\boldsymbol{P}_{\text {mask }}=\left(P_{\text {mask }}\left(f_{1}\right), P_{\text {mask }}\left(f_{2}\right), \cdots, P_{\text {mask }}\left(f_{K}\right)\right)$ 分别为 $K$ 个子 信道上的干扰容限, 则所有认知用户在信道 $f_{k}$ 上传输的 限制条件可以表式为:

$$
\sum_{i \in \Phi_{N}}\left\|\boldsymbol{w}_{i}^{(k)}\right\|^{2} \leq P_{\text {mask }}\left(f_{k}\right)
$$

在认知用户 $i$ 的接收端将其他认知用户发送的信息当 做干扰处理, 则在信道 $f_{k}$ 上认知用户 $i$ 的香农速率可以表 示为下式:

$$
R_{i}^{(k)}=\log \left(1+\boldsymbol{w}_{i}^{(k) H} \boldsymbol{h}_{i, i}^{(k)} C_{i}^{(k)-1} \boldsymbol{h}_{i, i}^{(k)} \boldsymbol{w}_{i}^{(k)}\right)
$$

其中, $\mathrm{C}_{i}^{(k)}$ 表示认知用户 $i$ 在信道 $f_{k}$ 上受到的噪声加 干扰, 其表示如下: 


$$
\mathrm{C}_{i}^{(k)}=1+\sum_{j \in \Phi_{N} \backslash\{i\}} \boldsymbol{h}_{i, j}^{(k)} \boldsymbol{w}_{j}^{(k)} \boldsymbol{w}_{j}^{(k) H} \boldsymbol{h}_{i, j}^{(k) H}
$$

由 (3) 式可得, 认知用户 $i$ 在 $K$ 个信道上的总传输 速率可以表示为:

$$
R_{i}=\sum_{k \in \Psi_{K}} R_{i}^{(k)}
$$

其中, 认知用户 $i$ 的传输速率 $R_{i}$ 不得低于门限速率 $c_{i}$, 即满足下式:

$$
R_{i} \geq c_{i}
$$

综上, 在MISO认知无线电网络中, 网络传输总功率 优化问题需要考虑以下三个约束条件: 每个认知用户传输 速率要大于接收端门限速率, 对授权用户的总干扰小于授 权容限和各个认知用户发射功率要小于最大发射功率。认 知用户可以通过合理的选择预编码向量, 使整个认知网络 的传输总功率最小。

定义 $\boldsymbol{W}_{i}=\left\{\boldsymbol{w}_{i}^{(1)}, \boldsymbol{w}_{i}^{(2)}, \cdots, \boldsymbol{w}_{i}^{(K)}\right\}$ 为认知用户 $i$ 在所有 $K$ 个信道上的预编码矩阵, 包含了 $K$ 个 $M \times 1$ 维的列向量, 定义 $\boldsymbol{W}=\left\{\boldsymbol{W}_{1}, \boldsymbol{W}_{2}, \cdots, \boldsymbol{W}_{N}\right\}$ 为各个认知用户的预编码矩阵, 包含了 $N$ 个 $M \times K$ 维的矩阵, 所考虑的最优化问题可以描 述为:

$$
\min _{W} \sum_{i \in \Phi_{N}} \sum_{k \in \Psi_{K}}\left\|\boldsymbol{w}_{i}^{(k)}\right\|^{2}
$$

s.t. $\quad \mathrm{Cl}: c_{i} \leq R_{i}, \forall i \in \Phi_{N}$

$\mathrm{C} 2:\left\|\boldsymbol{w}_{i}^{(k)}\right\|^{2} \leq P_{\text {mask }}\left(f_{k}\right), \forall k \in \Psi_{k}, \forall i \in \Phi_{N}$

$\mathrm{C} 3: \sum_{k \in \Psi_{K}}\left\|\boldsymbol{w}_{i}^{(k)}\right\|^{2} \leq P_{\max }$

如（7）式所示的最优化问题是NP-hard问题[13], 然 而求解NP-hard问题的算法复杂度往往较高, 为避免直接 求解, 可将该问题转化为非协作模型, 即通过构建单个认 知用户的最优化问题去求解各认知用户的功率优化问题, 从而得到各个用户的预编码矩阵。由于在认知网络中每个 用户在非协作模型中都是“自私的”，即各个认知用户在门 限速率和最大化发射功率的限制下, 都会尽可能地最大化 自身利益而不关心对其他认知用户造成的干扰，导致原来 传输质量较好的授权信道的信道质量会因多个认知用户 的同时占用而急剧下降。此外, 各认知用户为达到门限速 率要求会设法增加发射功率, 进而导致整个网络的功率消 耗急剧增加。为解决上述问题, 通过在非协作单认知用户 模型中引入价格因子来构建博恋模型, 进而合理的在各个 信道上分配功率, 最终实现网络传输总功率优化的目的。

认知用户 $i$ 的策略空间可定义为 $\boldsymbol{W}_{i}=\left\{\boldsymbol{w}_{i}^{(1)}, \boldsymbol{w}_{i}^{(2)}, \cdots, \boldsymbol{w}_{i}^{(K)}\right\}$, 其中 $\boldsymbol{W}_{i}$ 为一个 $M \times K$ 的矩阵, 包含了 $K$ 个 $M \times 1$ 维的向量。 网络中其他认知用户的效用函数具有与认知用户 $i$ 相近的表 示形式, 都可以表示为关于预编码矩阵的函数。网络中认知
用户 $i$ 以外的其他认知用户的策略选择可定义为 $\boldsymbol{W}_{-i}=\left(\boldsymbol{W}_{1}, \boldsymbol{W}_{2}, \cdots, \boldsymbol{W}_{i-1}, \boldsymbol{W}_{i+1}, \cdots, \boldsymbol{W}_{N}\right)$, 则认知用户 $i$ 的效用函数可 以表示为:

$$
U_{i}\left(\boldsymbol{W}_{i}, \boldsymbol{W}_{-i}\right)=-\sum_{k \in \Psi_{K}}\left\|\boldsymbol{w}_{i}^{(k)}\right\|^{2}
$$

（7）式所表示的最优化问题的纳什均衡解的存在性 取决于以下三个因素: 各认知用户通信速率需求下限、各 子信道上授权用户可以承受的干扰上限以及各认知用户 的发射功率上限。在非协作博弯模型中, 合理有效的价格 函数不仅能够有效地提高整个网络的效益, 还能够保证每 个认知用户合理地进行各自的策略选择, 从而达到整体网 络的传输总功率达到最小。

根据上述限制条件，结合（8）式中原认知用户 $i$ 的效 用函数，可构建如下所示的认知用户 $i$ 的博亦模型:

$$
\begin{array}{ll}
\max & U_{i}^{\prime}\left(\boldsymbol{W}_{i}, \boldsymbol{W}_{-i}\right) \\
\text { s.t. } & \mathrm{C} 1: R_{i, \min } \leq R_{i}, \forall i \in \Phi_{N} \\
& \mathrm{C} 2:\left\|\boldsymbol{w}_{i}^{(k)}\right\|^{2} \leq P_{\text {mask }}\left(f_{k}\right), \forall k \in \Psi_{k}, \forall i \in \Phi_{N} \\
& \mathrm{C} 3: \sum_{k \in \Psi_{K}}\left\|\boldsymbol{w}_{i}^{(k)}\right\|^{2} \leq P_{\max }
\end{array}
$$

其中, $U_{i}^{\prime}\left(\boldsymbol{W}_{i}, \boldsymbol{W}_{-i}\right)$ 为含有价格函数的认知用户 $i$ 的效 用函数，表达式如下:

$$
U_{i}^{\prime}\left(\boldsymbol{W}_{i}, \boldsymbol{W}_{-i}\right)=U_{i}\left(\boldsymbol{W}_{i}, \boldsymbol{W}_{-i}\right)-F_{i}\left(W_{i}\right)
$$

其中, $F_{i}\left(\mathbf{W}_{i}\right)$ 为认知用户 $i$ 的价格函数, 可以表示为 下式:

$$
F\left(\boldsymbol{W}_{i}\right)=\operatorname{tr}\left[\boldsymbol{W}_{i} \boldsymbol{A}_{i} \boldsymbol{W}_{i}^{H}\right]
$$

价格函数的设定要依赖于全局信道状态信息, 为降低 求解最优化问题的计算复杂度, 通常设定价格函数为线性 的。为了给出认知用户 $i$ 的价格函数 $F_{i}\left(W_{i}\right)$ 的表示形式, 需量化整个网络的干扰量, 即对每个认知用户接收端的噪 声加干扰协方差矩阵取迹, 进而得到整个网络的干扰函数 NIF为:

$$
N I F=K N\left(1+I_{p u}(k)\right) \operatorname{tr}(\mathrm{I})+\sum_{i \in \Phi_{N}} \operatorname{tr}\left[\boldsymbol{W}_{i}^{H} \times \boldsymbol{A}_{i} \times \boldsymbol{W}_{i}\right]
$$

其中 $\boldsymbol{A}_{i}$ 表示认知用户 $i$ 价格因子, 是一个维数为 $M \times M$ 的对角矩阵, $\boldsymbol{A}_{i}$ 对角线上第 $k$ 个元素为 $A_{i}^{(k)}$ 表示 认知用户 $i$ 在信道 $f_{k}$ 上的价格因子。NIF的函数值为一个 正数, 其单位和功率相同。

网络干扰函数NIF表达式中第一项表示来自授权用户 的干扰, 第二项表示整个网络中所有用户进行通信时总的 干扰。假设网络中各个认知用户都可以控制天线波束辐射 方向以避免对其他认知用户的通信造成干扰, 则网络中的 
干扰量主要来自于认知用户的同道干扰和邻道干扰, 为保 证认知用户能合理地在各信道上分配功率, 设定认知用户 $i$ 在信道 $f_{k}$ 上的价格因子 $A_{i}^{(k)}$ 的函数表达式如下:

$$
A_{i}^{(k)}=\sum_{j \in \Phi_{N} \backslash\{i\}} h_{j, i}^{(k)} h_{j, i}^{(k) H}
$$

（13）式中认知用户 $i$ 的价格因子取决于自身到邻近 认知用户接收端的信道增益矩阵。因此, 在实际网络中, 认知用户终端设备必须具备监听来自MAC层的信号包以获 取信道增益信息的能力。

综上, (9)式中的认知用户 $i$ 的博弯模型可被表示为:

$$
\begin{array}{ll}
\max & \left\{-\sum_{k \in \Psi_{K}} \operatorname{tr}\left(\boldsymbol{w}_{i}^{(k) H}\left[1+A_{i}^{(k)}\right] \boldsymbol{w}_{i}^{(k)}\right)\right\} \\
\text { s.t. } & \mathrm{C} 1: R_{i, \min } \leq R_{i}, \forall i \in \Phi_{N} \\
& \mathrm{C} 2:\left\|\boldsymbol{w}_{i}^{(k)}\right\|^{2} \leq P_{\operatorname{mask}}\left(f_{k}\right), \forall k \in \Psi_{k}, \forall i \in \Phi_{N} \\
& \mathrm{C} 3: \sum_{k \in \Psi_{K}}\left\|\boldsymbol{w}_{i}^{(k)}\right\|^{2} \leq P_{\max }
\end{array}
$$

在 (14) 式中, 因为矩阵 $\boldsymbol{A}_{i}$ 为半正定矩阵, 所以 $1+A_{i}^{(k)}$ 是一个正数, 同时该博亦模型中效用函数关于策略空间 $Q$ 的收玫性与未加入价格函数的（7）式最优化问题相同。

\section{2. 纳什均衡点求解}

针对 (14) 式所示的最优化问题, 提出采用改进的 Gauss-Seidel迭代 (即逐次松弛迭代) 算法进行求解。逐 次松弛迭代算法一般被用于线性方程组的迭代求解，是一 种经典的迭代方法, 具有较高的收玫速度。利用逐次松驰 迭代算法, 对已建立的博弯模型 (14) 式进行求解。根据 文献 [11] 直接计算 $(14)$ 式所需计算的变量个数为 $2 K M^{2}$, 远远大于其对偶问题中的变量个数 $K+2$, 同时 (14) 式 为凸最优化问题, 故可将该式转化为其对应的对偶问题, 然后利用逐次松驰迭代法求解该对偶问题，进而得到最优 的预编码矩阵。

可运用Hadamard不等式理论 [14]去分析和求解 (14) 式所对应的对偶问题:

$$
\mathrm{DP}: \min _{\left\{\lambda_{i}\right\}} \mathrm{D}\left(\lambda_{i}\right)
$$

其中 $\lambda_{i} \stackrel{\text { def }}{=}\left(\lambda_{i}^{(0)}, \lambda_{i}^{(1)}, \cdots, \lambda_{i}^{(K+1)}\right)$ 表示一个 $K+1$ 维的向 量, 其每个元素 $\lambda_{i}^{(k)}$ 为最优化方程 (14) 式所对应的拉格 朗日乘子。 $\mathrm{D}\left(\lambda_{u}\right)$ 表示对偶方程, 其定义如下:

$$
\mathrm{D}\left(\lambda_{i}\right)=\max _{\left\{\boldsymbol{w}_{i}^{(k)}, \forall k \in \Psi_{K}\right\}} L_{i}\left(\boldsymbol{w}_{i}^{(k)}, \lambda_{i}\right)
$$

其中 $L_{i}\left(\boldsymbol{w}_{i}^{(k)}, \lambda_{u}\right)$ 为最优化方程 (14) 式所对应的拉格 朗日函数, 可表示为:

$$
\begin{aligned}
L_{i}\left(\boldsymbol{w}_{i}^{(k)}, \lambda_{i}\right)= & -\sum_{k \in \Psi_{K}} \operatorname{tr}\left(\boldsymbol{w}_{i}^{(k) H}\left[1+\mathrm{A}_{i}^{k}\right] \boldsymbol{w}_{i}^{(k)}\right) \\
& -\lambda_{i}^{(0)}\left(R_{i, \min }-R_{i}\right) \\
& -\sum_{k \in \Psi_{K}} \lambda_{i}^{(k)}\left(\operatorname{tr}\left(\boldsymbol{w}_{i}^{(k) H} \boldsymbol{w}_{i}^{(k)}\right)-P_{\text {mask }}\left(f_{k}\right)\right) \\
& -\lambda_{i}^{(k+1)}\left(\sum_{k \in \Psi_{K}} \operatorname{tr}\left(\boldsymbol{w}_{i}^{(k) H} \boldsymbol{w}_{i}^{(k)}\right)-P_{\max }\right)
\end{aligned}
$$

最优化问题 (14) 式在纳什均衡点处的最优预编码向 量 $\boldsymbol{w}_{i}^{(k)}$ 是矩阵 $\boldsymbol{h}_{i, i}^{(k) H} \mathrm{C}_{i}^{(k)-1} h_{i, i}^{(k)}$ 的特征向量, 其对应的特征 根为 $1+\lambda_{i}^{(k)}+\lambda_{i}^{(\mathrm{K}+1)}+A_{i}^{(k)}$, 即有下式成立:

$\boldsymbol{h}_{i, i}^{(k) H} \mathrm{C}_{i}^{(k)-1} h_{i, i}^{(k)} \boldsymbol{w}_{i}^{(k)}=\left(1+\lambda_{i}^{(k)}+\lambda_{i}^{(\mathrm{K}+1)}+A_{i}^{(k)}\right) \boldsymbol{w}_{i}^{(k)}$

上式指出了在纳什均衡点处 $\boldsymbol{w}_{i}^{(k)}$ 所需要满足的约束 条件, 为求解 $\boldsymbol{w}_{i}^{(k)}$, 定义 $\boldsymbol{P}_{k}^{(i)}$ 为认知用户 $i$ 在信道 $k$ 上的 功率分配向量, $\hat{\boldsymbol{w}}_{i}^{(k)}=\boldsymbol{w}_{i}^{(k)} /\left\|\boldsymbol{w}_{i}^{(k)}\right\|$ 为认知用户 $i$ 的归一化 预编码矩阵。由于 $\boldsymbol{P}_{k}^{(i)}$ 和 $\hat{\boldsymbol{w}}_{i}^{(k)}$ 需满足 $\boldsymbol{w}_{i}^{(k)}=\boldsymbol{P}_{k}^{(i)} \hat{\boldsymbol{w}}_{i}^{(k)}$, 同 时 $\hat{\boldsymbol{w}}_{i}^{(k)}$ 为 $\boldsymbol{w}_{i}^{(k)}$ 的归一化预编码向量, 因此 $\hat{\boldsymbol{w}}_{i}^{(k)}$ 同样能够对 角化下面两个式子 [11]:

$$
\begin{aligned}
r_{i}^{(k)} & =\hat{\boldsymbol{w}}_{i}^{(k)}\left[\boldsymbol{h}_{i, i}^{(k) H} \mathrm{C}_{i}^{(k)-1} \boldsymbol{h}_{i, i}^{(k)}\right] \hat{\boldsymbol{w}}_{i}^{(k) H} \quad \text { and } \\
\Lambda_{i}^{(k)} & \left.=\hat{\boldsymbol{w}}_{i}^{(k)}\left[\mathrm{A}_{i}^{k}+1+\lambda_{i}^{K+1}+\lambda_{i}^{k}\right)\right] \hat{\boldsymbol{w}}_{i}^{(k) H}
\end{aligned}
$$

其中, $\boldsymbol{Y}_{i}^{(k)}$ 和 $\Lambda_{i}^{(k)}$ 为 $M \times M$ 维的对角矩阵。将上式代 入到 (17) 式中, 并对其求偏导可得 $P_{s, k}^{(i)}$ 的表达式如下:

$$
P_{s, k}^{i}=\max \left(0, \frac{\lambda_{i}^{(0)} \operatorname{diags}\left(\Upsilon_{i}^{(k)}\right)-\operatorname{diags}\left(\Lambda_{i}^{(k)}\right)}{\operatorname{diags}\left(Y_{i}^{(k)}\right) \operatorname{diags}\left(\Lambda_{i}^{(k)}\right)}\right)
$$

至此, 最优的辐射方向 $\hat{\boldsymbol{w}}_{i}^{(k)}$ 和最优的功率分配 $\boldsymbol{P}_{s, k}^{(i)}$ 都 已利用拉格朗日因子 $\lambda_{i}^{(k)}$ 的表示, 能够利用逐次松驰迭代 算法进行迭代求解。算法迭代流程如表1所示。

表1 算法流程。

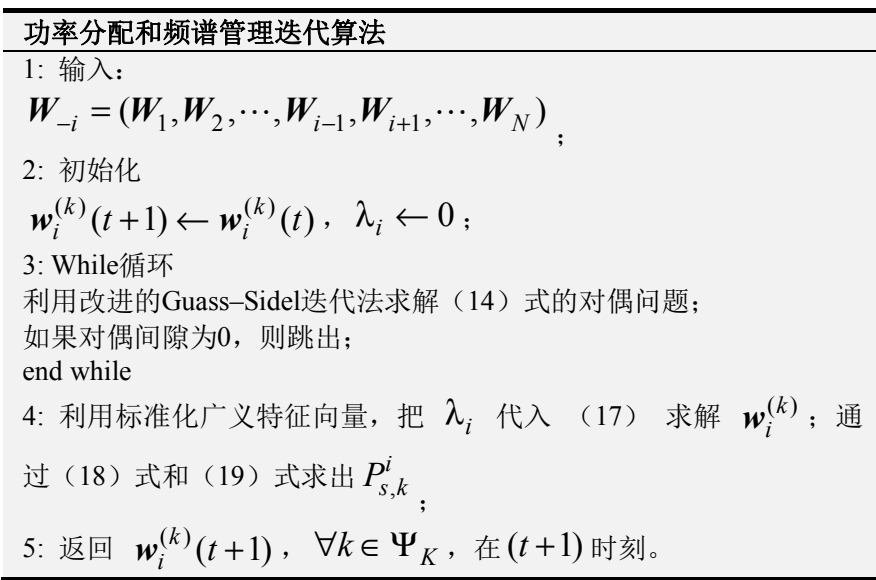




\section{4. 仿真及性能分析}

为了验证所提算法的有效性, 在此通过仿真实验给出 了所提算法在不同条件下的性能。在每个仿真实验中，仿 真结果都是经过 10 次运行得到的平均值。假设 $N$ 条认知用 户链路随机分布于 $100 \times 100$ 的认知无线电网络中, 网络中 有 $K=3$ 个可用的授权频段, 其中心频率分别为 $f_{1}=2.4 \mathrm{GHz}, f_{2}=2.7 \mathrm{GHz}, f_{3}=3 \mathrm{GHz}$, 三个频段都具 有 $60 \mathrm{MHz}$ 带宽。设每个认知用户的最大发射功率为 $P_{\text {max }}=2 \mathrm{~mW}$, 各信道干扰容限为 $P_{\text {mask }}=1.5 \mathrm{~mW}$, 信道为 平坦衰落信道, 自由空间衰减系数设为 3.5 , 逐次松驰迭 代的影响因子设为 1.25 , 噪声功率谱密度设为 $-174 \mathrm{dBm} / \mathrm{Hz}$ 。主用户各信道上的干扰限制设为 $-100 \mathrm{dBm} / \mathrm{Hz}$ 。

图2给出了所提算法在不同门限速率的限制条件下网 络传输总功率随认知用户数量变化的曲线。从图中可以看 出, 随着认知用户数的增加, 网络总传输总功率也在增加。 当认知用户数量一定时, 认知用户接收端的门限速率越高, 则网络总传输总功率越大。这是由于随着网络中认知用户 数量的增多, 认知用户之间的干扰也在加大, 系统为保证 认知用户的速率能到达通信所需的门限值, 发射端会适当 地增加发射功率。

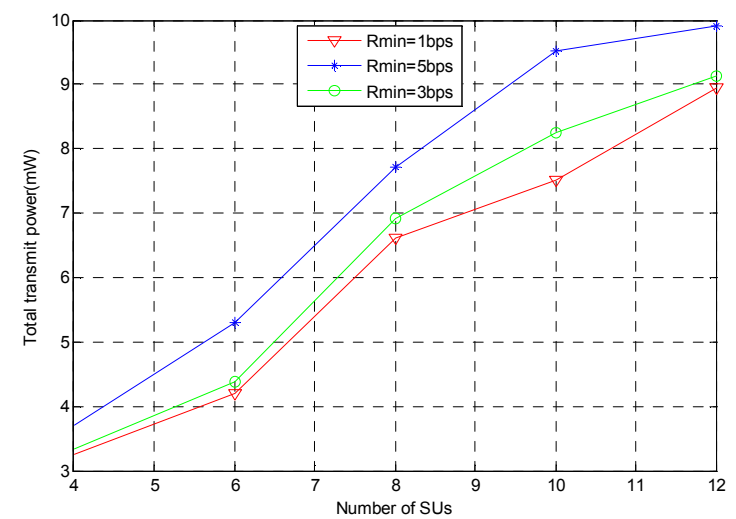

图2 不同认知用户数量条件下的网络传输总功率。

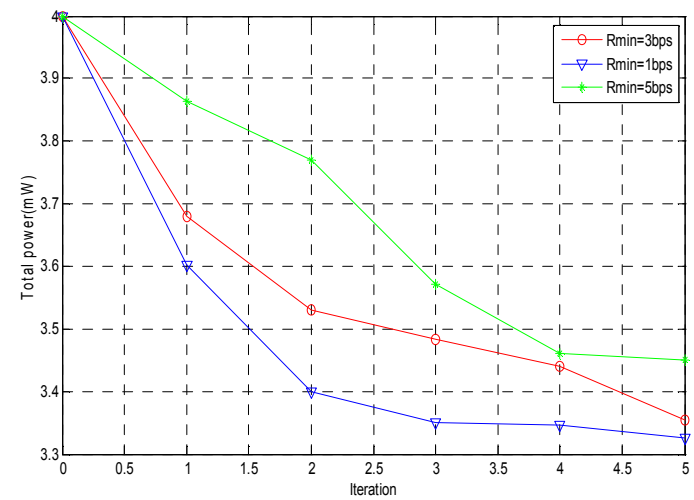

图3 不同迭代次数条件下的网络传输总功率。

图3给出了在不同的认知用户速率要求下网络传输总 功率随迭代次数变化的曲线图, 其中认知用户数目固定为 4。从图中可以看出, 当认知用户数固定时, 随着迭代次
数的增多, 网络传输总功率在不断降低。同时可以看出, 迭代次数越多, 算法结果越趋于稳定, 仿真结果证明了算 法的有效性。从图 3 可知, 所提算法由于联合空域和频域 进行求解, 提出了价格函数, 考虑了认知用户信道选择的 公平性, 明显地减小了网络传输总功率。在认知用户数量 固定, 认知用户接收端门限速率设置不同的情况下, 初始 网络传输总功率都为 $4 \mathrm{~mW}$, 迭代完成后得到的网络传输总 功率不同。从图中可以看出, 随着认知用户门限速率的增 加, 迭代完成得到的网络传输总功率会变大。

图4给出了网络传输总功率随认知用户数量变化的曲 线图。从图4可以看出, 随着认知用户数量增多, 网络传 输总功率不断增加, 但在认知用户数量相同的条件下, 没 有引入价格函数的算法获得的网络传输总功率要比所提 算法获得的网络传输总功率大, 即所提算法更好地实现了 网络传输总功率的优化。这是由于所提算法引入了合理的 价格因子, 有效地控制了认知用户之间的干扰, 在不同信 道上合理地为各个认知用户分配了功率, 不仅能够最小化 网络传输总功率, 而且有效地保证了不同认知用户的服务 质量需求。

图5给出了各个信道上的功率分配情况, 其中认知用 户数目为 4 , 信道个数为 3 。从图中可以看到, 所提算法在 各个信道上为认知用户均匀分配了功率资源, 而不含价格 函数的算法在第三个信道上却没有分配功率, 并且不含价 格函数的算法在第一个信道上分配的功率比其它信道的

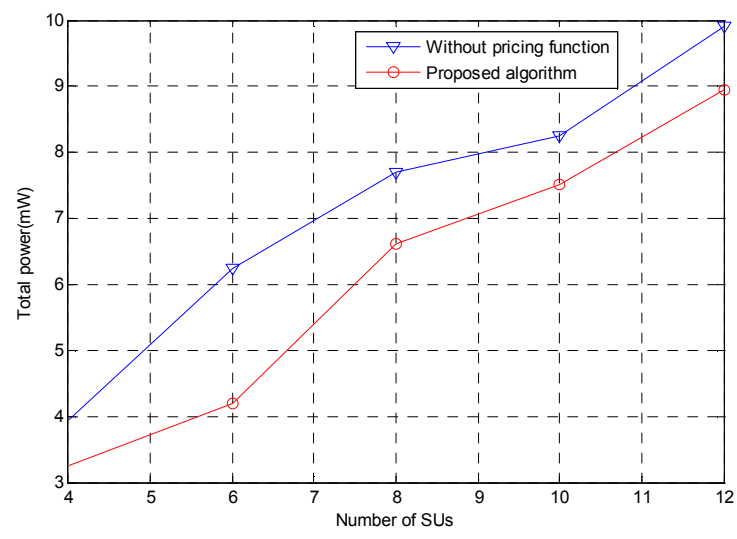

图4 经典算法与所提算法的功率比较。

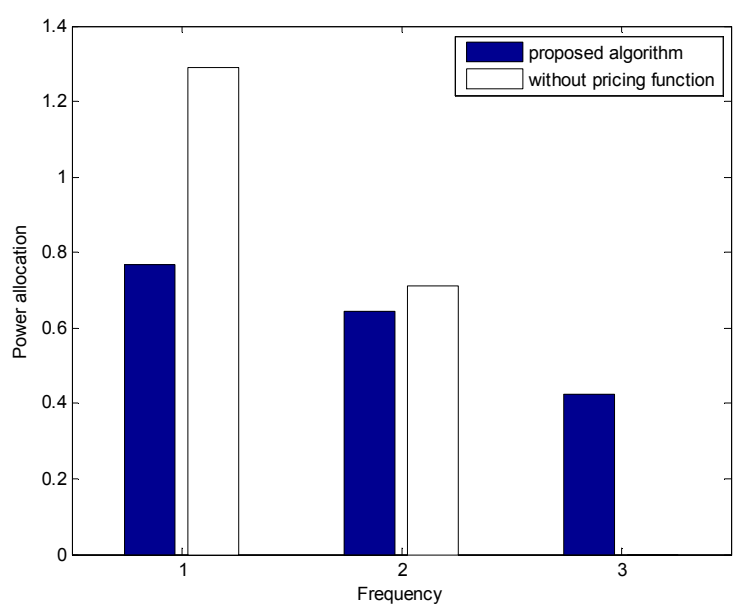

图5 两种算法在不同信道上的功率分配。 
大得多, 这是由于不含价格函数的算法只是根据目前信道 的状况给当前认知用户分配功率, 并且在条件较好的信道 上尽可能分配较多的功率, 没有考虑对其他用户造成的干 扰。所提算法通过引入价格函数, 导致在信道条件较好的 信道上分配较多的功率需要付出更高的代价, 从而有效地 控制了认知用户之间的干扰。在各个信道上均匀为认知用 户分配功率, 合理地利用了频谱资源, 使网络传输总功率 最小。

\section{5. 结论}

本文提出了一种基于博弯论的频谱资源空域重用算 法。该算法利用博弯论建立了频谱资源空域重用问题的博 弯模型, 然后基于认知用户的信道质量引入价格因子来保 证认知用户在各信道上进行功率分配的公平性。最后利用 逐次松弛迭代算法求解纳什均衡点, 获得了功率分配的最 优解。所提算法不仅能够使网络传输总功率最小化, 而且 能够保证认知用户的服务质量需求。仿真结果验证了所提 算法的有效性。

\section{致谢}

本文成果获得如下项目支持: 新世纪优秀人才支持计 划项目（NCET-13-0105）; 河北省高校百名优秀创新人 才支持计划项目（BR2-259）; 河北省自然科学基金项目 （No. F2016501139）; 中国高等教育博士研究生专项科 研基金（No.20130042110003）; 中央高校基本科研业务 费专项资金资助项目（No. N142302001, No.N162304002)。

\section{参考文献}

[1] Haykin S. Cognitive radio: brain-empowered wireless communications [J]. IEEE Journal Selected Areas in Communications, 2005, 23(2): 201-220.

[2] Etkin R, Parekh A, Tse D. Spectrum sharing for unlicensed bands [J]. IEEE Journal on Selected Areas in Communications, 2007, 25(3): 517-528.
[3] P. W. 登特. 从公共天线发射多个无线电信道频率信号的 发射机和方法: 中国, 00819007.3 [P]. 2006-07-26。

[4] Kolodzy. Next generation communications: Kichoff meeting [J]. Proc.Darpa.2001, (10): 3-26.

[5] Pan C, Su C, Ren $\mathrm{H}$, et al. An online algorithm of energy-efficient and interference suppression beamforming for cognitive MISO-OFDM interference channels [C]. IEEE International Conference on Wireless Communications \& Signal Processing (WCSP), 2013: 1-6.

[6] Dahrouj H, Yu W. Coordinated beamforming for the multicell multi-antenna wireless system [J]. IEEE Transactions on Wireless Communications, 2010, 9(5): 1748-1759.

[7] Nedic A, Ozdaglar A. Distributed subgradient methods for multi-agent optimization [J]. IEEE Transactions on Automatic Control, 2009, 54(1): 48-61.

[8] 詹德睿. 认知无线电中频谱共享技术研究 $[D]$. 北京邮电大 学, 2013。

[9] Nguyen D N, Krunz M. Price-based joint beamforming and spectrum management in multi-antenna cognitive radio networks [J]. IEEE Journal on Selected Areas in Communications, 2012, 30(11): 2295-2305.

[10] 刘秉瑞, 李乾. 基于认知无线电的空时域频谱共享研究 [J]. 移动通信, 2016, 40(12): 46-51。

[11] 吴帆, 郑趂哲. 基于博亦论的频谱动态管理研究 $[\mathrm{J}]$. 计算 机研究与发展, 2016, 53(1): 38-52。

[12] Dong H, Chun Y M, Yuan M. A game theory approach for self-coexistence analysis among IEEE 802.22 networks [C]. Communications and Signal Processing International Conference on Information, 2009: 1-5.

[13] Nguyen D N, Krunz M. Power minimization in MIMO cognitive networks using beamforming games [J]. IEEE Journal on Selected Areas in Communications, 2013, 31(5): 916-925.

[14] Scutari G, Palomar D P, Barbarossa S. Asynchronous iterative water-filling for Gaussian frequency-selective interference channels [J]. IEEE Transactions on Information Theory, 2008, 54(7): 2868-2878. 